\title{
LE TRAITEMENT DE L'ESPACE DANS TROIS CONTES DE MARCEL SCHWOB : VERS LE FANTASTIQUE
}

Kévin FRANÇOIS

Université de Varsovie

\begin{abstract}
En): Despite the difficulties that may arise when we try to define the fantastic, it is possible to adopt a specific theoretical point of view enabling us to mobilise this genre. Once such a position was adopted, we tried, through this study, to analyse how Marcel Schwob uses space in three of his tales: "Le Roi au masque d'or", "Les Embaumeuses" and "Pays bleu", to create what could be called "fantastic effects ". Those spaces have been observed through the theory of Roman Ingarden, a polish phenomenologist, which helped us to estimate how much they can be modulated in order to create different reading effects.
\end{abstract}

Key words (En): mental space ; fantastic ; fantastic effects ; vagueness ; Marcel Schwob ; Roman Ingarden ; phenomenology

Mots clés (Fr): Espace mental; fantastique; effet fantastique; indétermination; Marcel Schwob ; Roman Ingarden ; phénoménologie

\section{L'espace littéraire et l'effet fantastique : considérations générales}

L'espace est une partie inhérente à toute œuvre artistique, qui peut se décliner sous bien des aspects. L'être humain se meut et vit dans un espace précis, mais peut être appelé à en traverser d'autres, à travers l'art par exemple. Chaque domaine artistique prend place ou crée un espace particulier, lié ou connecté aux autres de différentes manières, mais toujours défini par ses limites. En effet, cette conception de limites, de frontières, est intrinsèquement liée à la notion d'espace, elle permet de les distinguer entre eux afin de pouvoir les définir et les étudier. Au cours de cette étude, nous allons nous pencher sur l'espace littéraire dont les limites ne sont pourtant pas si aisément saisissables. En effet, nous pourrions d'abord penser que ce dernier est tout simplement limité par le langage et les mots de l'auteur. Mais cela reviendrait à considérer que l'œuvre littéraire n'est que le produit de celui qui écrit. Or, Wolfgang ISER l'a bien souligné dans sa théorie de la réception, une œuvre est formée par la relation créée entre le lecteur et l'auteur: "on peut dire que l'œuvre littéraire à deux pôles : le pôle artistique et le pôle esthétique : Le pôle artistique se réfère au texte produit par l'auteur tandis que le pôle esthétique se rapporte à la concrétisation réalisée par le lecteur » (1976:48). Un texte ne peut donc se réduire à l'un ou l'autre et il n'est pas possible d'impunément évincer la personne qui à chaque lecture recrée ce que l'encre de l'auteur n'a en rien figé. Les limites d'une production littéraire semblent alors devenir incommensurables, relevant de la personnalité et de l'imagination de chaque personne lisant le livre. Néanmoins, ce n'est pas pour autant que nous allons professer à la manière de Roland Barthes la mort de l'auteur : l'auteur conserve tout de même un statut de guide, il est celui qui met sur la voie et qui invite le lecteur au voyage. Il est celui qui pose les fondations de cet espace mental et qui à ce niveau fait des choix, prend des décisions pour tracer, à sa manière, le chemin qu'il veut que son lecteur suive. 
Il dispose d'un grand pouvoir sur cet espace créé, mais pour cela un profond travail est réalisé. Il y a donc au sein d'une œuvre confrontation entre au moins deux espaces mentaux : celui de l'auteur et celui du lecteur. Nous nous en rendons donc bien compte, la question de l'espace au sein d'une œuvre littéraire n'est pas si évidente et ne peut se limiter à un seul parti. Cela se complexifie d'autant plus si nous étudions la façon dont chaque genre traite cette question, mais au cours de cette étude, nous allons surtout nous pencher sur un auteur en particulier: Marcel Schwob, auteur français du XIXe siècle, souvent rattaché au mouvement symboliste, et sur trois de ses contes : «Les Embaumeuses », «Le Pays bleu », et «Le Roi au masque d'or», tous trois tirés de son recueil Le Roi au masque d'or (1892), et tous trois pouvant être d'une certaine manière rattachés au fantastique. À travers ces trois récits, nous allons nous efforcer de comprendre de quelle manière l'auteur jongle avec les différents espaces de l'œuvre afin de créer ou de renforcer des effets fantastiques.

Mais avant tout, il est évident que nous ne pouvons innocemment avancer ce terme de fantastique sans nous pencher plus en avant dessus. Afin de le définir, nous pourrions nous référer à la définition de Tzvetan Todorov qui a su faire autorité :

Dans un monde qui est bien le nôtre, celui que nous connaissons, sans diables, sylphides, ni vampires, se produit un événement qui ne peut s'expliquer par les lois de ce même monde familier. Celui qui perçoit l'événement doit opter pour l'une des deux solutions possibles : ou bien il s'agit d'une illusion des sens, d'un produit de l'imagination et les lois du monde restent alors ce qu'elles sont ; ou bien l'événement a véritablement eu lieu, il est partie intégrante de la réalité, mais alors cette réalité est régie par des lois inconnues de nous. [...] Le fantastique occupe le temps de cette incertitude ; dès qu'on choisit l'une ou l'autre réponse, on quitte le fantastique pour entrer dans un genre voisin, l'étrange ou le merveilleux (ToDOROv, $1970: 29$ )

Tzvetan Todorov nous présente donc le fantastique comme un fil ténu, une danse au bord de l'abîme où le lecteur perd ses repères, ne comprend plus ce monde décrit qui semblait pourtant le sien. Il ne réside d'ailleurs selon l'auteur que dans ce court instant et est perdu à jamais une fois ressenti : une deuxième lecture et le charme fantastique du texte s'est évanoui (TODOROV 1970 : 95). Certains aspects de cette définition peuvent donc nous sembler assez restrictifs car elle ne tient compte que d'un ressenti éphémère de la part du lecteur et écarte l'ambiance créée par l'auteur et ses effets continus sur l'affect de celui qui lit. Rachel BOUVET déclare de son côté que «l'on ne se trouve jamais de but en blanc dans un récit fantastique face à un événement étrange : on l'attend avec impatience. Les indices de l'étrange glanés tout au long du récit ne font qu'exacerber l'attente du lecteur. Enfin, le récit fantastique met la compréhension du lecteur à rude épreuve » (1992:43). Il existe donc plusieurs présentations du fantastique qui est une notion difficile à définir précisément, et pour laquelle nous allons donc nous rattacher à un de ses traits décisifs : l'implication et la participation du lecteur, partie intégrante de toutes les définitions relevées : celle de « Todorov, souvent considérée comme étant l'une des plus importantes [définitions du fantastique] met au premier plan l'hésitation du lecteur entre une explication naturelle et une explication surnaturelle. Pour Lovecraft, c'est le sentiment de peur ressenti par le lecteur qui sert de critère pour affirmer l'appartenance d'un récit au genre fantastique. Caillois, quant à lui, insiste 
sur l' « impression d'étrangeté irréductible » qui se dégage de ces récits. » (BOUVET, 1992 : 41) C'est donc chez le lecteur que l'effet se crée, comme le font remarquer tous deux Louis VAX : «l'homme qui lit une histoire à faire peur participe de la peur du personnage menacé » (1965: 1), et Tzvetan TODOROV : «Le fantastique implique donc une intégration du lecteur au monde des personnages, il se définit par la perception ambigüe qu'a le lecteur même des événements rapportés » (1970:35). C'est donc en pensant aux sensations que va éprouver le lecteur que peut être rédigé un récit fantastique. Ainsi le fantastique est une notion subjective, entièrement dépendante d'une façon de penser et de ressentir, elle-même découlant de nombreux facteurs comme l'époque, l'appartenance à un groupe social, etc. Comme le déclare Louis VAX: "Chacun se forge une image du monde, chacun possède une connaissance plus ou moins obscure du possible et de l'impossible, du normal et du monstrueux. Qu' une fêlure apparaisse en ce monde, et le fantastique surgit » (1965 : 169), et nous pouvons donc retenir que l'effet fantastique survient à travers une rupture dans un mode de pensée, dans un moment où la raison ne semble plus avoir tous ses droits (VAX, $1965: 14)$. L'auteur à travers son récit crée un univers, un espace, dans lequel il sait provoquer des ruptures afin de susciter le sentiment de fantastique chez le lecteur.

Ces effets fantastiques peuvent être produits de plusieurs manières et sont à la discrétion de l'auteur. Nous n'allons pas au cours de cette étude tous les analyser, mais nous concentrer sur la manière dont Marcel Schwob a su moduler l'espace dans les trois contes cités afin de créer ou renforcer de tels effets. Mais avant de réaliser cette étude, il nous faut présenter les travaux sur lesquels nous nous sommes basés pour modéliser l'espace mis en place par l'auteur. Dans son livre, L'œuvre d'art littéraire (1983), Roman Ingarden, phénoménologue polonais, s'est efforcé de mettre en exergue ce qui distingue l'œuvre littéraire dans son essence. Au cours de son raisonnement, l'auteur fait la distinction entre trois types d'espaces : l'espace réel, l'espace figuré, et l'espace représenté. L'espace réel est celui de l'auteur, du lecteur, celui que nous côtoyons quotidiennement et dans lequel nous faisons notre vie. L'espace figuré est :

un espace propre, qui par essence fait partie du monde «réel » figuré. En un certain sens, il est apparenté à tous ces espaces [espaces réel, idéal, géométrique], dans la mesure où il présente une structure qui permet encore de l'appeler «espace », quand bien même avoir cette structure n'est qu'un avoir fictif et simulé. D'après sa structure, il est relativement le plus proche de l'espace objectif réel (resp. de l'espace d'orientation perceptuel) (INGARDEN, 1983 : 191).

C'est donc de manière simplifiée celui qui est mis en place et décrit par l'auteur dans son récit. L'espace représenté est celui que les écrits de l'auteur font naître dans l'esprit du lecteur. Cette représentation est une forme d'appropriation personnalisée, différente pour chacun, mais toujours guidée. Ingarden relève à travers ces trois cas que l'essence de l'espace en général est d'être sans discontinuités. Cette affirmation nous semble relever de l'évidence en ce qui concerne l'espace réel, mais demande plus de délicatesse d'esprit en ce qui concerne les espaces figuré et représenté. Cela tient surtout du fait que l'espace figuré rentre dans la catégorie des « objets figurés », l'ensemble des choses et personnes décrites par l'auteur, et que ces objets figurés sont soutenus par ce que l'auteur nomme des 
« lieux d'indétermination». Ce sont ces lieux d'indétermination qui permettent la continuité de ces deux espaces à l'aide d'une participation majeure du lecteur qui partiellement inconsciemment comble et peuple de manière personnelle les vides laissés par les mots de l'auteur. Nous pouvons relever pour les besoins de cette étude deux types de lieux d'indétermination: il y a les lieux d'indétermination qui permettent la continuité de l'espace figuré et donc de l'espace représenté, mais il y a aussi les lieux d'indétermination inhérents à l'espace figuré. Dans le premier cas, nous pouvons donner l'exemple dans «Les Embaumeuses» du narrateur qui raconte son voyage jusqu'en Libye, passant par « les neuf cercles de sables divers qui entourent l'Éthiopie»(VIBERT, 2009: 335). Schwob ne décrit pas dans les détails le chemin emprunté pour lier un pays à l'autre, mais automatiquement ces deux espaces sont reliés dans notre esprit. Ils ne prennent pas la forme d'îlots perdus dans du vide sur lesquels le narrateur se téléporterait de l'un à l'autre. Dans le deuxième cas, cela tient à ce qu'Ingarden appelle la «teneur » des objets figurés qui vient se calquer et fusionner avec la description de l'auteur, conférant à l'objet figuré des propriétés de l'objet réel dont il est issu. Par exemple, le conte «Le Roi au masque d'or » débute ainsi : «Le roi masqué d'or se dressa du trône noir où il était assis depuis des heures » (VIBERT, 2009 : 302). À la simple lecture de cette phrase, nous attachons aux mots «trône » et «roi » toute une dimension qui peuple immédiatement notre espace représenté. Cela tient de la «teneur », évidemment, mais aussi de notre imagination qui habille ce roi ou ce trône, le place dans une salle, un château, ou un ensemble référentiel dépendant de l'expérience que nous avons de ces mots. C'est donc à travers ces lieux d'indétermination que les espaces littéraires dépassent les seules limites du langage et des mots de l'auteur. Comme le souligne Ingarden :

Lorsqu'il crée, l'auteur n'est d'abord lié que par l'exigence d'écrire une œuvre intelligible et qui forme un ensemble cohérent. Il opère avec des phrases et des connexions de phrases, et de ce fait, il est lié par toutes les lois qui relèvent de l'essence de la phrase et des relations de phrases. Mais en ce qui concerne la teneur des « objets » figurés dans l'œuvre, elle peut en principe, dans une large mesure, être soumise à l'arbitraire, et en particulier être formée sans souci de savoir si elle ressemble, resp. ne ressemble pas, aux «objets » qui nous sont connus par expérience (INGARDEN, $1983: 255$ ).

L'auteur endosse donc à travers ses écrits le rôle de guide qui offre, dans son propre univers, une voie au lecteur et à son imagination.

Cette théorie de l'espace littéraire est très intéressante dans le cadre de notre étude sur le fantastique de Marcel Schwob, et d'autant plus lorsque cela se corrobore avec les propos de Louis VAX sur l'espace fantastique qui est pour lui « une variété de l'espace vécu » (1965: 196), passée par le prisme de l'imagination et des peurs de notre inconscient. En prenant l'exemple d'un homme qui marche dans la forêt la nuit, il déclare :

Objectivement, il ne fait rien que traverser un lieu circonscrit de l'espace, à une heure tardive ; subjectivement, il est captif d'un espace et d'un temps nés de sa peur. [...] Les choses ont cessé d'exister pour elles-mêmes, elles n'existent plus que dans la mesure où elles sont au service de la menace. [...] Assurément, pour la conscience épouvantée, la représentation de 
l'espace objectif n'est pas totalement anéantie. Mais le monde cesse d'avoir une réalité pleine. (VAX, 1965 : 196-197)

Les effets fantastiques prennent toute leur puissance dans l'espace représenté du lecteur, dans lequel il s'est laissé guider par l'espace figuré de l'auteur. Il est de plus un effet récurrent, mis en place par Marcel Schwob, contribuant à renforcer l'effet fantastique dans «Les Embaumeuses », «Le Pays Bleu», et «Le Roi au masque d'or ». Cet effet tient de la possession ou plutôt de la dépossession de l'espace du narrateur, du personnage principal, par l'auteur du récit : au fur et à mesure que le récit se déroule, Schwob parvient à priver le personnage principal de l'espace qu'il découvre, qu'il essaie d'occuper. Il nourrit les lieux d'indétermination et contribue à remettre en cause la vision de l'espace perçu, comme si nous étions condamnés à y être étrangers et donc à ne jamais être capables d'en percer les secrets, le sens, s'il $\mathrm{y}$ en a un.

Nous percevons donc bien qu'une forme de modulation de l'espace littéraire par l'auteur peut participer à la production d'effets fantastiques. Voyons la manière de procéder de Marcel Schwob dans trois de ses contes.

\section{L'espace schwobien et les théories de Roman Ingarden}

\section{« Les Embaumeuses »}

Ce conte est narré par un voyageur grec, accompagné de son frère, Ophélion, qui voyagent ensemble dans le désert couleur sang de Libye. Au cours du huitième jour passé à traverser cette étendue cruelle, ils aperçoivent « des coupoles blanches de petite dimension disposées en cercle» (VIBERT, 2009 : 336), vers lesquelles ils s'acheminent pour être accueillis par une femme vivant dans l'une d'elles. Ils prennent le souper avec leur hôte puis vont dormir. Lorsque le narrateur s'éveille au cours de la nuit, il ne trouve plus son frère à ses côtés et décide de le chercher dans le village. Il se rend ainsi compte que ce lieu est une «cité des embaumeuses» (VIBERT, 2009 : 339), qu'il décide de fuir avec son frère dès le jour venu. Alors qu'il s'apprête à rentrer dans la coupole où ils ont tous deux été accueillis, il lui semble entendre son frère Ophélion passant du temps avec une femme. À ce moment-là leur hôte arrive et le narrateur plonge dans un profond sommeil qui ne prend fin qu'au matin, où il se rend compte que son frère est allongé à ses côtés mais terriblement malade. Ce dernier meurt le soir même et le narrateur s'endort sous le poids de son chagrin constatant à son réveil que son hôte et celle qui semble être sa sœur jumelle sont en train d'embaumer le corps de son frère. Il pense à une histoire de luxure et de jalousie, et s'enfuit sans demander son reste.

Nous percevons dans ce récit plusieurs éléments fantastiques tenant du suspense, de la soudaineté et de l'incompréhension. Une rupture est bien créée avec un élément qui échappe à la compréhension du narrateur, et par extension à celle du lecteur, pour laquelle il ne peut qu'émettre des hypothèses. Il est très intéressant de remarquer que ce récit est rédigé à la première personne avec un narrateur qui est de plus anonyme, seul son frère est nommé. Comme le souligne Todorov, «la première personne "racontante" est celle qui permet le plus aisément l'identification du lecteur au personnage, puisque, comme on sait, le pronom "je" appartient à tous. 
En outre, pour faciliter l'identification, le narrateur sera "un homme moyen"» (TODOROV, 1970: 89), mais surtout elle facilite ce que Louis VAX nomme la « contamination » (1965: 124) c'est-à-dire une participation affective du lecteur à ce qui arrive au narrateur. Cette contamination contribue aussi à l'intégration du lecteur au sein de l'espace figuré :

Si nous voulons alors saisir, à la lecture, le monde figuré exactement comme il est figuré, nous devons pour ainsi dire nous transporter de manière fictive dans le centre d'orientation figuré, nous promener in fictione avec le personnage en question à travers l'espace figuré. Une bonne figuration nous contraint par elle-même à le faire (INGARDEN, 1983 : 197-198),

ce qui lui permet d'être à son tour ce qu'Husserl nomme «le point zéro de l'orientation » (INGARDEN, 1983: 197) à partir duquel l'espace est porté à figuration.

Observons de quelle manière Marcel Schwob parvient à moduler l'espace de son récit afin de créer des effets fantastiques. Tout d'abord, l'espace figuré choisi fait référence à des espaces réels, mais en y ajoutant une pointe de merveilleux : «Qu'il y ait encore en Libye, sur les confins de l'Éthiopie où vivent les hommes très vieux et très sages, des sorcelleries plus mystérieuses que celles des magiciennes de Thessalie, je ne puis en douter» (VIBERT, 2009: 334), « Nous avions traversé, mon frère Ophélion et moi, les neufs cercles de sables divers qui entourent l'Éthiopie » (VIBERT, 2009 : 335). Ces noms de Libye et d'Éthiopie nous sont connus, le lecteur peut y avoir été ou vouloir s'y rendre, ils font partie de l'espace réel. Mais Schwob, en mentionnant cette magie et ces cercles, en transfigurant ces lieux, participe à la création d'un lieu d'indétermination secondaire renforcé. Le lecteur perd ses repères, il parvient à situer ce lieu mentionné à l'aide de sa teneur, mais il se rend compte qu'il ne le connaît plus, qu'il lui échappe. Cette rupture créée entre l'espace réel et l'espace figuré participe à la création d'une ambiance fantastique, nourrie par les lieux d'indétermination. Mais ce qui renforce surtout une telle ambiance est l'approximation avec laquelle est décrit l'espace figuré. Le point zéro d'orientation de ce conte est le narrateur, c'est à travers lui que nous découvrons la Libye et son désert rouge. Comme le souligne Sabrina Granger, cette découverte nous est rendue de manière bien floue : « le narrateur n'a de cesse de souligner les failles de son discours : tournures négatives et modalisateurs se multiplient. En outre, la présence des thèmes des apparences trompeuses et de l'obscurité contribuent à disqualifier l'instance énonciatrice : témoin peu fiable, il est possible qu'il ait été dupé par ses sens »(VIBERT, 2009: 334). En effet, observons quelques passages où le narrateur décrit l'espace dans lequel il se meut (les termes d'approximation sont soulignés pour les besoins de l'étude) : « Il y a des dunes terrestres qui, dans le lointain paraissent glauques »(VIBERT, 2009: 335), « quand nous nous approchâmes, l'obscurité était grande » (VIBERT, 2009 : 336), «L'ouverture de ces portes était sombre (VIBERT, 2009: 336), «L'entrée était obscure ainsi que la salle ronde sous la coupole » (VIBERT, $2009: 337$ ), « sans qu'il nous fût possible de voir, à la faible lueur de la lampe » (VIBERT, 2009 : 337), « je sortis dans la nuit, et il me sembla que j'entendais » (VIBERT, 2009 : 338). Il apparaît ainsi de manière évidente que le narrateur est lui-même perdu dans cet espace, et 
par le phénomène de contamination, le lecteur se trouve perdu à son tour, sans réellement savoir comment imaginer cet espace décrit qui recèle de zones d'ombre. Ainsi, en recourant à un narrateur interne peu fiable pour faire découvrir son univers au lecteur à travers l'espace figuré, Marcel Schwob guide l'espace représenté dans une incertitude propice au fantastique. Il y a donc bien une modulation de l'espace permettant à l'auteur d'insérer du fantastique.

Mais il est un dernier point qui concerne l'espace et qu'il est important d'étudier pour sa participation à un effet fantastique. Le lecteur, à travers le narrateur, fait, comme nous l'avons mentionné, l'expérience d'une dépossession de l'espace. Ce phénomène renforce la perte de repères, le monde semble s'échapper et le personnage principal comme le lecteur se retrouvent désarmés, impuissants. Cela est permis dans un premier temps par cette approximation avec laquelle le narrateur saisit les lieux qui l'entourent, il ne peut pleinement se les approprier, ni pleinement se situer. La femme qui les invite, lui et son frère, dans sa coupole, semble quant à elle maîtriser totalement cet espace : elle disparaît et apparaît soudainement à sa guise sans que le narrateur ne puisse distinguer par où elle passe. Par la suite, alors que le narrateur comprend finalement où il se trouve, qu'il décide même de rentrer « sous notre [marque d'appropriation] coupole » (VIBERT, 2009 : 339) et y découvre un escalier souterrain, la dépossession survient avec cette femme qui réapparaît « sous la coupole [redevenue un lieu lointain] », d'une ouverture qu'il ne fait que supposer, et qui semble l'endormir. Après ce somme, il ne parvient même plus à retrouver l'escalier précédemment révélé. Ainsi, nous pouvons affirmer que cette dépossession qui suit l'appropriation de l'espace par les découvertes du narrateur (même illusoires), est un phénomène voulu par Marcel Schwob induisant un effet fantastique. Comme le relève Louis VAX : «Le mystère n'est pas dans les choses, mais dans la lutte confuse d'une raison s'efforçant de reconquérir un monde qui menaçait de lui échapper» (1965: 106). Dans ce récit, le narrateur n'a pas su s'emparer de cet espace, il n'est pas parvenu à percer ses secrets, il ne peut émettre que des doutes quant à ce qui est arrivé à son frère. Toutes ces incertitudes permettent la formation d'un espace figuré plein de lieux d'indétermination propices à laisser flous les espaces représentés. Ainsi, en jonglant entre les différentes modulations de l'espace et le rapport que son narrateur établit avec, Schwob crée un effet fantastique, remettant en cause les repères qu'essaie d'établir le lecteur.

\section{«Le Pays bleu »}

Cette histoire raconte la rencontre du narrateur et d'une petite fille appelée Maïe un soir d'hiver. Cette enfant l'invite dans son logis où il découvre sa misère et décide de l'aider, elle et son compagnon Michel. Le narrateur rend donc visite à Maïe de manière régulière et celle-ci lui raconte comment elle est arrivée en ville, comment elle se débrouille, mais surtout, l'histoire du « Pays bleu », un paysage issu d'une pièce de théâtre dont elle croit en la réalité et où elle veut se rendre. Mais alors que Maïe sort peu à peu de la misère, notamment grâce au soutien du narrateur, elle devient de plus en plus triste et morose, jusqu'à ce que l'hiver se termine enfin et que le narrateur, en lui rendant visite un soir, découvre cette note laissée par l'enfant : «Bonsoir, mon chez-moi. Maïe et Michel sont partis pour le Pays bleu » (VIBERT, 2009 : 468). 
Nous retrouvons dans ce récit le narrateur interne, anonyme, et le lecteur est ainsi, à l'aide du phénomène de la contamination, une nouvelle fois au point zéro de l'orientation. Il ne peut découvrir l'espace figuré qu'à travers les yeux du personnage racontant l'histoire. Cet espace est d'ailleurs laissé dans une forme de brouillard d'incertitude, mais de manière différente : le narrateur n'est pas capable de situer l'endroit où il se trouve : «Dans une ville de province que je ne saurais plus retrouver, les rues montantes sont vieilles et les maisons vêtues d'ardoise » (VIBERT, 2009 : 463), même s’il reste précis et imagé dans les descriptions qu'il en fait, contrairement au narrateur précédent. Ce qui renforce le côté mystérieux de l'espace de ce conte, c'est ce phénomène de possession dont nous avons parlé dans la partie précédente. Dans « Le Pays Bleu », après avoir partiellement décrit la ville où la rencontre avec la fillette a eu lieu, le narrateur n'a de cesse ensuite de se laisser guider par cette dernière. L'espace figuré devient celui de Maïe et le narrateur n'y est qu'invité, à tel point que la jeune fille semble même exercer un certain pouvoir à l'endroit où elle se trouve. Elle personnalise sa chambre et les objets qui y sont placés, elle crée un espace à part, plein de magie parce qu'elle l'a souhaité ainsi. Tout au long du récit, le lecteur, par l'intermédiaire du narrateur, ne peut que subir l'espace et son mystère. Maïe l'a entièrement investi de sa présence, elle l'a envahi et va même, par ses injonctions, jusqu'à en chasser Michel qui ne fait que de brèves apparitions, tout de suite rappelé à l'ordre. Schwob semble avoir conféré à sa « nymphette » le pouvoir de refaire le monde, comme le souligne Michel Viegnes dans sa préface au conte : «L'appartement misérable, avec son mobilier délabré ce que l'on appellerait aujourd'hui un squat - est transfiguré par le seul pouvoir de la parole» (VIBERT, 2009: 461). Nous pouvons ainsi la rapprocher de l'embaumeuse du récit étudié précédemment, le narrateur semble être totalement dépendant du bon vouloir de son hôte. L'auteur nous place donc ici dans un espace qui nous échappe une nouvelle fois, que nous ne pouvons réellement rationaliser. Un doute subsiste quant aux pouvoirs réels de cette petite fille car justement nous avons été placés, d'une certaine façon, à sa merci. Sa parole, sa façon de s'adresser à ce qui l'entoure crée des lieux d'indétermination. Encore une fois, un effet fantastique a été permis par la modulation de l'espace et son rendu. Nous nous rendons donc bien compte des possibles stratégies que l'auteur peut mettre en place pour influer sur les espaces mentaux.

\section{« Le Roi au masque d'or »}

Ce conte nous intéresse particulièrement car il peut s'apparenter sous certains aspects à un récit initiatique et ainsi ne ressemble pas aux deux autres étudiés précédemment. Le narrateur n'y est pas interne et la contamination se présente de manière plus empathique. Dans cette histoire, nous suivons les pas d'un roi anonyme dont la figure, comme celle de tous ses sujets, est cachée par un masque. Le sien est d'or et «à l'imitation du roi décharné, les femmes, les bouffons et les prêtres avaient d'immuables figures d'argent, de fer, de cuivre, de bois et d'étoffe » (VIBERT, 2009 : 302-303). Le souverain accepte un jour de recevoir un vieillard à la vue trouble et au visage découvert qui lui annonce qu'il ne vit que dans un monde d'illusions et d'apparences, qu'il ne connaît rien de ses sujets. Suite à ce reproche, le roi décide de sortir de son palais en toute humilité et rencontre en sortant d'une 
forêt une belle jeune fille pour qui il enlève son masque afin de pouvoir l'embrasser. À la vue de ce visage découvert, la belle s'enfuit en criant, provoquant le désarroi du roi qui s'en va contempler son reflet dans le cours d'un fleuve. Il se rend ainsi compte qu'il semble atteint de la lèpre et que son masque n'avait pour but que de cacher sa malédiction héréditaire. Il rentre alors au château confondre ses pères à travers leur portrait, et sa cour, avant de procéder à son énucléation pour se protéger de l'apparence fallacieuse des choses. Suite à cet acte il s'en va parcourir la campagne avant de rencontrer une jeune lépreuse, que le roi aveugle prend pour une éleveuse de brebis à des clochettes qu'elle porte pour signaler son passage, avec qui il décide de se rendre à la cité des Misérables. En vue de leur destination, le roi meurt en annonçant que tout ce qu'il aurait souhaité était de poser ses lèvres sur celles de sa compagne, mais qu'il s'en était retenu de peur de la souiller de sa maladie. Ces mots provoquent le désespoir de la jeune fille qui ne comprend pas ce qu'a pu se reprocher cet être au visage si pur alors qu'elle était atteinte de la maladie qu'il décriait. C'est sur ses pleurs qu'apparaît de nouveau le vieillard du début du conte qui semble expliquer la guérison miraculeuse du roi par son geste de sacrifice.

$\mathrm{Ce}$ récit peut sembler à première vue moins mystérieux que «Les Embaumeuses » et «Le Pays bleu » et son rattachement au registre fantastique s'avère bien plus délicat, résidant tout de même selon nous dans le « Sans doute » du vieux mendiant, prononcé à la fin du conte pour tenter d'expliquer une guérison d'apparence miraculeuse alors que la jeune lépreuse pleure sur le corps du roi : «Sans doute le sang de son cœur qui avait jailli par ses yeux avait guéri sa maladie. » (VIBERT, 2009 : 316) Ce « sans doute » est une locution particulière car il induit le doute justement, et peut aussi se traduire par «probablement». Une explication est donc donnée, mais peu satisfaisante car elle ne peut pas même totalement se rattacher au merveilleux, à cause de cette locution qui nous incite à remettre les choses en perspective. Y a-t-il vraiment guérison miraculeuse et donc intervention merveilleuse, ou nous faut-il chercher une raison rationnelle à cette guérison ? Le visage couvert depuis l'enfance par un masque peut en effet se trouver d'horrible aspect, sans pour autant être touché par la lèpre, maladie dont le roi s'autodiagnostique à partir de souvenirs et d'un reflet dans l'eau (VIBERT, 2009 : 308). Marcel Schwob laisse donc son lecteur hésiter et la modulation de l'espace dans ce texte, sans pour autant créer d'effet fantastique renforce tout de même l'atmosphère propre à ce genre. Le château de ce roi est particulier par ses traditions, mais les descriptions que nous en donne l'auteur peuvent se référer à tout château que n'importe quel lecteur aurait pu visiter. Le roi semble tout à fait maître de son domaine, jusqu'à ce que les paroles du vieillard viennent troubler son esprit. Alors, à la recherche de la vérité, le roi se voit contraint de quitter cet espace qui lui est familier, en partie car «dans toute l'étendue de la résidence royale, il n'y avait pas un miroir » (VIBERT, 2009: 306). Et c'est justement dans ce nouvel espace, celui qui correspond au dehors, à l'extérieur, que survient la rupture provoquée par la terrible vérité. Bien sûr des doutes s'étaient déjà immiscés au moment du discours du mendiant, mais c'est en dehors du château que le roi perd tout repère. Suite à cette découverte, le roi se lance dans une course pour confronter tout ce qu'il pensait connaître, il traverse son palais avec vélocité, jusqu'à commettre un sacrifice : son énucléation. Cet acte est hautement symbolique car suite à celui-ci, il se retrouve à 
son tour, comme les narrateurs des deux contes précédemment étudiés, mais d'une manière différente, dépossédé de son espace, jusqu'à se heurter aux murailles ou se blesser sur les haies (VIBERT, 2009 : 314). Cette dépossession n'est pas à mettre au niveau des deux autres car elle ne crée pas par elle-même le mystère : dans «Les Embaumeuses » le narrateur comme le lecteur semble perdre tout pouvoir alors que l'hôte reprend possession de son habitat, dans «Le Pays bleu » le narrateur comme le lecteur ne sont plus que des objets disposés selon le bon vouloir de Maïe. Mais dans «Le Roi au masque d'or », cette dépossession vaut refuge, c'est pour échapper au monde trompeur des apparences que le roi se plonge « dans cette plaine sombre des pensées » (VIBERT, 2009 : 314). Ainsi, cette modulation de l'espace par Schwob ne crée pas d'effet fantastique à proprement dit, elle soutient une atmosphère et une rupture mais n'en est en aucun cas l'incarnation. Il n'y a pas dans ce récit de culture de lieu d'indétermination, ils n'interviennent que comme conséquence à la limite des mots et du langage, mais ne sont pas provoqués pour créer de l'étrange. Malgré cela, nous nous rendons bien compte que Schwob donne un rôle primordial à l'espace, et qu'il est important de se pencher sur la possession de celui-ci.

\section{Conclusion}

Nous avons donc pu au cours de cette étude observer à travers trois contes de quelle manière Marcel Schwob joue sur les espaces mentaux pour créer ou renforcer des effets fantastiques. L'analyse du dernier récit, «Le Roi au masque d'or », nous a permis de nous rendre compte que cette modulation des espaces modélisés par Roman Ingarden n'est pas une nécessité dans tous les récits fantastiques, mais que revient chez Schwob cette problématique de la possession/dépossession de l'espace qui permet de créer une atmosphère semblant échapper au narrateur et donc au lecteur. Le déplacement de ces personnages autour de qui l'histoire se fait permet de créer une forme de passivité chez celui qui lit, qui devient ainsi plus perméable à tout effet fantastique. Cela se corrobore ainsi avec une remarque de Louis Vax selon qui : «Pour être prenant, le fantastique doit sourdre du monde et non jaillir de l'esprit de l'auteur» (VAX, 1965 : 227). Nous nous rendons donc bien compte que l'espace a un rôle primordial dans l'œuvre littéraire et qu'il se révèle être un formidable lien entre l'auteur et le lecteur. Il serait intéressant à cet effet de se pencher sur d'autres contes fantastiques où l'espace semble détenir un rôle majeur comme « La chute de la Maison Usher » d'Edgar Allan Poe, où d'entrée le narrateur semble subir une influence insidieuse de cette bâtisse (POE, 2008 : 132) et de son environnement. À quel point ce phénomène de possession/dépossession entre-t-il de nouveau en jeu pour renforcer un effet fantastique ? Pourrait-il s'appliquer de manière encore plus étendue à toute modulation de l'espace?

\section{BIBLIOGRAPHIE}

BOUVET Rachel (1992), «La lecture et ses troubles: analyse d'un conte fantastique », in Tangence, $\mathrm{n}^{\circ} 36$, p. 41-51.

INGARDEN Roman (1983), L'auvre d'art littéraire, Lausanne, L'âge d'homme.

ISER Wolfgang (1976), L'acte de lecture. Théorie de l'effet esthétique, Bruxelles, Pierre Mardaga. 
POE Edgar Allan (1974), Nouvelles histoires extraordinaires, Paris, Gallimard.

SCHWOB Marcel (1892). Le Roi au masque d'or, dans VIBERT Bertrand (éd. présentée par) (2009), Contes symbolistes, vol.1, Grenoble, Ellug.

TODOROV Tzvetan (1970), Introduction à la littérature fantastique. Paris, Editions du Seuil.

VAX Louis (1965), La séduction de l'étrange, Paris, Presses Universitaires de France. 